\title{
Articulación y percepción de /t/ en el habla de adultos mayores edéntulos y portadores de prótesis dentales totales
}

\section{Articulation and perception of the / $/ \mathrm{t}$ phoneme in a speech corpus of edentulous elderly adults wearing dental prostheses}

\author{
EMMANUEL ADONAY JARQUÍN CASAS ${ }^{a}$ \\ FERNANDA LÓPEZ-ESCOBEDO ${ }^{b}$
}

\author{
a Universidad Nacional Autónoma de México, Facultad de Filosofía y Letras. México. \\ Correo electrónico: adonayjcasas@hotmail.com \\ ${ }^{b}$ Universidad Nacional Autónoma de México, Ciencia Forense, Facultad de Medicina. México. \\ Correo electrónico: flopeze@unam.mx
}

En este trabajo se presentan las principales realizaciones del fonema / $/$ / en un corpus de habla de adultos de la tercera edad, edéntulos y portadores de prótesis dental, de la Ciudad de México. Se observaron realizaciones oclusivas, fricativas y africadas del fonema $/ \mathrm{t} / \mathrm{con}$ una duración que va desde los 23 milisegundos (ms) hasta los $271 \mathrm{~ms}$, además de variaciones en la duración de la segunda fase que va desde los $5 \mathrm{~ms}$ a los $92 \mathrm{~ms}$. Asimismo, se llevó a cabo un análisis sobre la pertinencia de los rasgos acústicos de este fonema mediante una serie de test de percepción auditiva. Los resultados mostraron que el fonema de estudio se identificó en la mayoría de los casos, a pesar de que las características acústicas prototípicas se modificaron o elidieron. Resultó de especial relevancia que la disminución en la duración total del estímulo no afectó la percepción de /t/.

Palabras claves: rasgos distintivos, fonética perceptiva, fonema dental oclusivo sordo, habla de adultos mayores, prótesis dental.

In this paper we present the main realizations of the / $t /$ phoneme in a speech corpus of Mexico City elderly adults with edentulism and wearing dental prostheses. Plosive, fricative and affricate realizations of the /t / phoneme with durations ranging from 23 milliseconds (ms) up to $271 \mathrm{~ms}$, as well as second phase durations spanning $5 \mathrm{~ms}$ to $92 \mathrm{~ms}$ were observed. An analysis of the relevance of the acoustic features of this phoneme through a series of auditory perception tests revealed that, 
in most cases, /t/ was identified despite the modification or elision of the prototypical acoustic characteristics. It was particularly relevant that the decrease in the total duration of the stimulus did not affect the perception of $/ \mathrm{t} /$.

Key words: distinctive features, speech perception, voiceless dental plosive phoneme, elderly adults voice, dental prostheses.

\section{INTRODUCCIÓN}

El estudio de la fonoarticulación en adultos mayores edéntulos y portadores de prótesis dentales ha sido escaso y en muchas ocasiones poco específico. La fonoarticulación de esta población puede afectarse por tres situaciones principales: el proceso natural de envejecimiento, el edentulismo, que es la pérdida de uno o todos los órganos dentarios, y el uso de prótesis dentales totales. El edentulismo puede dificultar la articulación de los fonemas vocálicos y consonánticos debido a los cambios anatómicos que genera. Las prótesis dentales totales suelen generar alteraciones en la cavidad oral y en la articulación de diversos fonemas consonánticos debido a la inclinación de los incisivos prostéticos (Runte et al. 2001), a la ausencia de las rugas palatinas (Kong y Hasen 2008) o al grosor de la base acrílica en la región palatal (Seifert et al. 1999). Estas afectaciones se presentan aun cuando una de las finalidades principales de las prótesis es facilitar la fonoarticulación. Comúnmente, estas alteraciones desaparecen tras un periodo de adaptación al nuevo entorno creado por la prótesis, aunque existen casos en los que el paciente no puede habituarse a las nuevas dimensiones de la cavidad oral (Kong y Hasen 2008), por lo que la dificultad articulatoria persiste.

Martínez Contreras (2013) señala que, en el caso de los fonemas oclusivos, especialmente en el fonema / $/$, la mala articulación con prótesis parciales se debe a un excesivo grosor en la región palatal anterior, aunque no describe en qué consiste la mala articulación de dicho fonema. En el caso de los retenedores, se tiene noticia de que pueden afectar el rasgo sonoro [t-d] y grave [t-p], debido a los cambios que generan en el entorno de la cavidad oral (Martínez et al. 2006). No obstante, no se cuenta con un registro de cómo es modificado el fonema /t/ del español mexicano en el plano articulatorio.

En lo que respecta al plano perceptivo, el estudio de la percepción de los fonemas oclusivos se ha centrado en determinar cuál es la característica principal para la distinción entre este tipo de fonemas. Tradicionalmente se ha propuesto la sonoridad como el rasgo distintivo de las series de oclusivas /p/, /t/, /k/ y /b/, /d/, /g/, tal como lo expone Quilis (1999) y la Real Academia Española (2011). En contraste, se ha propuesto la tensión como el rasgo que caracteriza estas dos series de oclusivas.

Lisker (1957) planteó que la duración de la oclusión distingue estas dos series de fonemas al comprobar mediante una serie de test perceptivos que una oclusiva con una duración menor a $70 \mathrm{~ms}$ es interpretada como un fonema sonoro, y con una duración mayor a $80 \mathrm{~ms}$ es interpretada como un fonema sordo. El experimento de Lisker consistió 
en insertar fragmentos de silencio en la oclusiva de la palabra rupee, en rangos de $10 \mathrm{~ms}$, obteniendo estímulos con una duración que iba desde los 40 ms hasta los 150 ms.

Liberman et al. (1961) llevaron a cabo un experimento similar con la palabra rapid. Estos autores utilizaron estímulos sintetizados con la finalidad de que otras características acústicas como la sonoridad o las transiciones vocálicas no afectaran el análisis de la tensión. Los resultados que obtuvieron coinciden con los de Lisker (1957) al identificar dos categorías definidas. El estímulo es percibido como un fonema sonoro cuando presenta una duración de menos de $50 \mathrm{~ms}$, como un sordo con una duración entre 80 y $100 \mathrm{~ms}$, y proponen una tercera categoría con una duración superior a los $120 \mathrm{~ms}$ que corresponde a los fonemas geminados.

Álvarez (1978), a su vez, confirma los resultados de Liberman et al. (1961) mediante una serie de test perceptivos utilizando estímulos sintéticos con la palabra gamblingy observa que con una duración entre 40 y 70 ms las oclusivas se interpretan como sonoras, de 90 a 170 ms como sordas y con más de 200 ms como geminadas.

Ya en el ámbito hispánico, Martínez Celdrán (1993) retoma la metodología de estos autores y mediante una serie de test perceptivos de identificación y de discriminación auditiva ${ }^{1}$ obtiene tres categorías a partir de la duración de la oclusión: de 26 a $61 \mathrm{~ms}$ son sonoras, de 70 a $140 \mathrm{~ms}$ son sordas, y de los $149 \mathrm{~ms}$ en adelante son geminadas, aun cuando esta última categoría no es fonológicamente pertinente en el español. Es así como Martínez Celdrán (1993) plantea tres categorías basadas en el rasgo tenso para los fonemas oclusivos del español: flojas, tensas e hipertensas.

Martínez Celdrán (1991) propone otros argumentos que le dan relevancia fonológica a la tensión en el español al señalar que dicho rasgo puede crear contrastes entre distintos tipos de obstruyentes, pues un espacio de silencio que sustituye la primera mitad de un fonema fricativo puede ser interpretado como un fonema oclusivo.

Con respecto al papel que tiene la tensión en la distinción sobre los fonemas oclusivos, Quilis (1999) y la RAE (2011 \$7.1e) sostienen que este rasgo tiene parámetros acústicos discutibles, además de que es redundante en el sistema fonológico del español, pues la sonoridad es la que tiene mayor relevancia fonológica. Además, no se ha determinado un correlato articulatorio específico para la tensión. Ibabe et al. (2019), en la búsqueda de dicho correlato, analizaron por medio de resonancias magnéticas la serie $\left[\mathrm{k}-\mathrm{g}-\mathrm{\gamma}_{\mathrm{T}}-\mathrm{x}\right]$, encontrando evidencias de que existe una relación entre la tensión y la superficie de contacto de los órganos articuladores. Planas et al. (2020), mediante las técnicas de palatografía y electromiografía, confirmaron desde un enfoque articulatorio que las oclusivas sonoras presentan una menor tensión en la articulación con respecto a las oclusivas sordas /d-t/.

En el plano perceptivo, gran parte de los estudios de los fonemas oclusivos se ha centrado en la distinción entre los grupos /p/, /t/, /k/ y /b/, /d/, /g/. No obstante, en la población de estudio, adultos mayores portadores de prótesis dentales, los cambios

\footnotetext{
${ }^{1}$ En los test de identificación auditiva los informantes escogieron entre las opciones $b, p$ o $p p$, tras escuchar el estímulo auditivo; mientras que en los test de discriminación los informantes escucharon dos estímulos distintos e indicaron si se trataba del mismo estímulo o si había distinción entre ellos.
} 
anatómicos en la cavidad oral pueden generar una gran variación en la articulación de los fonemas oclusivos, hecho que interviene directamente en la percepción de éstos. Dichos cambios pueden afectar tanto los rasgos de sonoridad o tensión, como los de modo de articulación: oclusivo, fricativo o africado.

Así, el objetivo de este estudio consiste en analizar las características del fonema / $\mathrm{t} /$ en una población no prototípica que se ve en la necesidad de adaptar la producción del sonido y que, a pesar de los cambios en su articulación, logra que se perciba como tal. Para ello, es necesario, primero, describir las principales realizaciones del fonema $/ \mathrm{t} / \mathrm{en}$ el habla de los adultos mayores edéntulos y portadores de prótesis dentales. Se parte de la hipótesis de que el fonema / $\mathrm{t} /$ tiene una alta variabilidad en el corpus de estudio, además de que el uso de prótesis dentales genera cambios importantes en la producción de este fonema en comparación con la del adulto mayor edéntulo.

Para analizar la forma en la que estos cambios fonoarticulatorios son interpretados a nivel perceptivo se llevaron a cabo test de identificación, en los que el informante tenía que dar nombre a la sílaba que creía estar escuchando; y test de discriminación, en los que el informante tenía que elegir entre alguna de las tres opciones que se le proporcionaban en una planilla de papel. Se partió de la hipótesis de que este fonema es reconocido por el interlocutor a pesar de que sus características prototípicas, tensión, explosión y duración del fonema, sufren cambios a nivel articulatorio.

\section{Metodología}

El material sonoro que se utilizó para esta investigación forma parte del Corpus Registro de Voz en el Adulto Mayor, el cual fue creado por el Grupo de Ingeniería Lingüística del Instituto de Ingeniería de la Universidad Nacional Autónoma de México (UNAM)². Está compuesto por un conjunto de grabaciones de la voz de trece adultos mayores, hombres y mujeres, portadores de prótesis dentales totales quienes acudieron a la Clínica de Admisión de la Facultad de Odontología de la UNAM, que es el lugar de primer contacto para la recepción, registro, valoración y distribución de los pacientes que reciben atención odontológica por parte de estudiantes de la Facultad. A los pacientes portadores de prótesis dental se les invitó a participar en el Corpus, y aquellos que dieron su consentimiento fueron grabados en un salón con poco ruido dentro de la Clínica y utilizando una grabadora digital. Las muestras de voz se grabaron en un formato de alta calidad y consistieron en una conversación entre el informante y la entrevistadora. La duración de las grabaciones es en promedio de 16 minutos. El subcorpus seleccionado para este trabajo está constituido

\footnotetext{
${ }^{2}$ La recopilación del corpus fue realizada por Blanca Flor Ramos Gamboa, estudiante de la Facultad de Odontología de la UNAM y becaria del Grupo de Ingeniería Lingüística. La finalidad de este corpus era contar con una colección de audios que permitieran hacer un estudio de la voz en el adulto mayor portador de prótesis dental, desde un punto de vista odontológico.
} 
por las grabaciones de seis informantes, 3 hombres y 3 mujeres; con un rango de edad que va de los 63 a los 78 ańos y cuya formación académica es de nivel básico. Respecto a las características odontológicas, todos son edéntulos totales, a excepción de una de ellas, quien aún conservaba los incisivos inferiores.

Para el análisis acústico se buscó que el fonema dental oclusivo sordo /t/ estuviera precedido por un fonema alveolar fricativo sordo /s/ y seguido de una vocal, es decir, en el contexto stV. Este contexto fue estudiado por Martínez Celdrán (1991) en un experimento perceptivo para determinar si la fase de silencio es suficiente para que un fonema oclusivo sea interpretado como tal, aun cuando otras características como la explosión o el VOT no estén presentes durante la articulación.

El etiquetado del corpus fue realizado con el programa Praat en su versión para Windows y los parámetros acústicos que se tomaron en cuenta fueron las fases articulatorias presentes: tensión, para los espacios de silencio correspondientes a la oclusión; explosión, para la barra de energía breve característica de esta fase; y la fricción, que se aprecia en el espectrograma como una concentración de energía turbulenta en las frecuencias mayores a $3000 \mathrm{~Hz}$ y cuya intensidad es menor en comparación con un fonema fricativo.

Una vez segmentadas cada una de las fases de $/ \mathrm{t} /$, se analizaron temporalmente por separado, así como la duración total del segmento. El análisis fue realizado con la ayuda de un script de Praat ${ }^{3}$ que permitió obtener de manera automática la duración de cada etiqueta.

La organización de los datos se basó en la realización del fonema /t/, por tal motivo se obtuvieron tres tipos de articulación según sus fases articulatorias. En primer lugar, se encontró una realización oclusiva con relajación explosiva con las fases tensión-explosión; una realización africada con las fases tensión-fricción, y una realización fricativa con la fase fricción.

En segundo lugar, se realizó una clasificación a partir de la duración de la segunda fase articulatoria. Esta clasificación se fundamentó en los datos aportados por Quilis (1999) y Martínez Celdrán y Fernández Planas (2007), quienes mencionan que la duración de la segunda fase en las oclusivas explosivas tiene una duración máxima de $10 \mathrm{~ms}$, en las oclusivas aspiradas tiene una duración máxima de $30 \mathrm{~ms}$ y en las africadas una mínima de $50 \mathrm{~ms}$. Para la clasificación se tomaron en cuenta tres rangos temporales: breve, de 0 a 20 ms; medio, de 21 a 40 ms; y largo, de $41 \mathrm{~ms}$ en adelante. Esta división temporal tuvo como finalidad que los rangos propuestos por los autores se encontraran a la mitad de los rangos que se proponen en este trabajo, de forma que se amplía la distancia entre los mismos y se evitan las zonas de cruce entre dichos rangos. Además, se obtienen rangos temporales con la misma duración en tramas de $20 \mathrm{~ms}$.

Por último, los resultados se organizaron según la duración total del fonema y se tomó en cuenta la descripción que aporta Martínez Celdrán (1993) de los fonemas oclusivos. Este autor señala que la duración caracteriza el tipo de oclusiva en cuestión: un rango sonoro tiene una duración menor de $69 \mathrm{~ms}$, un rango sordo tiene entre 70 y $140 \mathrm{~ms}$, y un geminado supera los $141 \mathrm{~ms}$.

${ }^{3}$ El script utilizado se obtuvo de la página: http://stel.ub.edu/labfon/es/scripts-de-praat 
Para el análisis perceptivo se llevaron a cabo test de identificación, en los que el informante tenía que dar nombre a la sílaba que creía estar escuchando; y test de discriminación, en los que el informante tenía que elegir entre alguna de las tres opciones que se le proporcionaban en una planilla de papel. En ambos casos, los estímulos se reprodujeron tres veces.

Se realizaron tres test de discriminación y tres de identificación en los que se iba cambiando la característica acústica que se quería poner a prueba. En total se realizaron seis test perceptivos con el diseño que se muestra en el cuadro 1. En el caso de los estímulos señuelo, todos presentaban una articulación prototípica ${ }^{4}$.

Cada test estaba formado por diez estímulos en total, seis del fonema / t/ con distintas realizaciones y cuatro del fonema señuelo correspondiente. Además, otro aspecto que se evaluó fue si el tipo de grabación de la que se tomaron los estímulos, con o sin prótesis, afectaba la percepción del fonema de estudio 5 .

Cuadro 1. Diseño de los test perceptivos

\begin{tabular}{|c|c|c|c|c|c|c|}
\hline Test & $\begin{array}{c}\text { Fases articulatorias } \\
\text { de } / \mathrm{t} /\end{array}$ & Tipo de test & $\begin{array}{c}\text { Característica } \\
\text { acústica observada }\end{array}$ & $\begin{array}{c}\text { Tipo de } \\
\text { grabación }\end{array}$ & $\begin{array}{c}\text { Fonema } \\
\text { señuelo }\end{array}$ \\
\hline 001 & Tensión-explosión & Identificación & $\begin{array}{c}\text { Duración total del } \\
\text { segmento }\end{array}$ & Con prótesis & /d/ \\
\hline 002 & Tensión-explosión & Discriminación & $\begin{array}{c}\text { Duración total del } \\
\text { segmento }\end{array}$ & Edéntulo & $/ \mathrm{d} /$ \\
\hline 003 & $\begin{array}{c}\text { Tensión- } \\
\text { explosión }\end{array}$ & $\begin{array}{c}\text { Tensión- } \\
\text { fricción }\end{array}$ & Identificación & $\begin{array}{c}\text { Duración y } \\
\text { articulación de la } \\
\text { segunda fase }\end{array}$ & Con prótesis & $/ \mathrm{t} /$ \\
\hline 005 & $\begin{array}{c}\text { Tensión- } \\
\text { explosión }\end{array}$ & $\begin{array}{l}\text { Tensión- } \\
\text { fricción }\end{array}$ & Discriminación & $\begin{array}{c}\text { Duración y } \\
\text { articulación de la } \\
\text { segunda fase }\end{array}$ & Edéntulo & $/ \mathrm{t} /$ \\
\hline 006 & Fricción & Identificación & $\begin{array}{c}\text { Fricción y duración } \\
\text { total del segmento }\end{array}$ & Con prótesis & $/ \mathrm{s} /$ \\
\hline
\end{tabular}

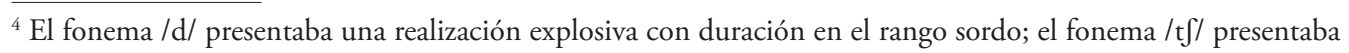
una realización africada con una duración total en el rango sordo y una duración de la segunda fase superior a los $50 \mathrm{~ms}$; el fonema /s/ presentaba una realización fricativa con una duración en el rango sordo.

${ }^{5}$ En una segunda ronda experimental se intercambiaron los estímulos de los test de identificación para que fueran utilizados en los test de discriminación y viceversa. Esto tuvo como finalidad verificar que el diseńo de los test fuera adecuado, además de observar si el hecho de que los estímulos provinieran de grabaciones con o sin prótesis tendría alguna influencia en la percepción de éstos. En la sección de resultados de este trabajo únicamente se presentaron los datos obtenidos en la primera de las rondas experimentales, ya que esta segunda ronda experimental únicamente se realizó como prueba control. 
Los estímulos auditivos fueron segmentados con la ayuda del programa Praat. Se seleccionó la sílaba y se separó del resto de la grabación, sin ningún tipo de compresión en el audio. Esto permitió que el informante únicamente contara con la información aportada por las fases articulatorias para el reconocimiento del fonema/t/. Así también fue posible controlar otros parámetros fonéticos como el contexto sonoro en el que se encontraba la sílaba, lo que evitó que el informante infiriera la sílaba correcta mediante este tipo de información.

Los test fueron aplicados a treinta estudiantes de la licenciatura en Lengua y Literaturas Hispánicas de la Facultad de Filosofía y Letras de la UNAM. En ningún caso tenían conocimiento previo de la finalidad de los test, únicamente se les pidió que siguieran las instrucciones correspondientes: nombrar o marcar cada uno de los estímulos que escuchaban. Cada test fue presentado con un dispositivo móvil tipo tablet compatible con formato WAV y unos auriculares de diadema con atenuador de ruido de almohadilla. Los test fueron realizados de manera continua en el orden descrito anteriormente, e individualmente.

\section{Resultados}

\subsection{Resultados del análisis acústico}

En total, se encontraron diecinueve variaciones del fonema /t/ en el corpus de estudio, donde la articulación prototípica: oclusiva con explosión breve y en el rango sordo, únicamente representa el $8.8 \%$ de las realizaciones de /t/ (véanse las negritas del cuadro 2).

Cuadro 2. Porcentaje de ocurrencia de las distintas realizaciones de / $\mathrm{t} /$ en el corpus

\begin{tabular}{|c|c|c|c|}
\hline Fases articulatorias & $\begin{array}{c}\text { Rango de duración } \\
\text { total del fonema }\end{array}$ & $\begin{array}{c}\text { Rango de duración de } \\
\text { la segunda fase }\end{array}$ & $\begin{array}{c}\text { Porcentaje de real- } \\
\text { ización en el corpus }\end{array}$ \\
\hline Tensión-fricción & Sordo & Media & $18.19 \%$ \\
\hline Fricción & Sordo & - & $14.58 \%$ \\
\hline Tensión-explosión & Sordo & Media & $13.18 \%$ \\
\hline Fricción & Sonoro & - & $13.50 \%$ \\
\hline Tensión-explosión & Sordo & Breve & $\mathbf{8 . 8 0} \%$ \\
\hline Tensión-explosión & Geminado & Media & $8.80 \%$ \\
\hline Tensión-fricción & Sordo & Larga & $6.39 \%$ \\
\hline Tensión-explosión & Geminado & Breve & $3.69 \%$ \\
\hline Tensión-fricción & Geminado & Media & $2.98 \%$ \\
\hline Tensión-explosión & Geminado & Larga & $2.63 \%$ \\
\hline
\end{tabular}




\begin{tabular}{|c|c|c|c|}
\hline Tensión-fricción & Sordo & Breve & $2.46 \%$ \\
\hline Tensión-explosión & Sordo & Larga & $1.93 \%$ \\
\hline Tensión-fricción & Sonoro & Media & $1.58 \%$ \\
\hline Tensión-fricción & Geminado & Larga & $1.05 \%$ \\
\hline Tensión-explosión & Sonoro & Breve & $0.07 \%$ \\
\hline Tensión-explosión & Sonoro & Media & $0.07 \%$ \\
\hline Tensión-fricción & Geminado & Breve & $0.05 \%$ \\
\hline Tensión-fricción & Sonoro & Breve & $0.03 \%$ \\
\hline Fricción & Geminado & - & $0.02 \%$ \\
\hline
\end{tabular}

Las demás variaciones de / $\mathrm{t} /$ corresponden a la combinación de las variables de duración total del fonema, tipo de articulación y duración de la segunda fase, así como a las fases articulatorias presentes. En el cuadro 2 se puede apreciar que los fonemas sordos y geminados ocupan las primeras posiciones por porcentaje de realización. La única variante del grupo sonoro que está en las primeras posiciones corresponde a una articulación fricativa, la cual tiene uno de los niveles de variación más altos en el corpus de estudio, pues no conserva ninguno de los rasgos característicos del fonema.

\subsubsection{Fases articulatorias del fonema /t/}

Los resultados indican que solo el 39\% de las realizaciones de /t/ cuentan con las fases tensión-explosión, en tanto que el $61 \%$ presenta algún elemento fricativo. Además, se puede apreciar en el cuadro 3 que el uso de prótesis facilita las fricciones, mientras que el edentulismo propicia una segunda fase explosiva.

Cuadro 3. Tipos de articulación del fonema /t/ según sus fases articulatorias

\begin{tabular}{|l|c|c|c|c|c|c|}
\hline & \multicolumn{2}{|c|}{ Edéntulo } & \multicolumn{2}{c|}{ Con prótesis } & \multicolumn{2}{c|}{ Total } \\
\cline { 2 - 7 } & $\begin{array}{c}\text { Porcentaje de } \\
\text { realización }\end{array}$ & $\begin{array}{c}\text { Duración } \\
\text { media }\end{array}$ & $\begin{array}{c}\text { Porcentaje de } \\
\text { realización }\end{array}$ & $\begin{array}{c}\text { Duración } \\
\text { media }\end{array}$ & $\begin{array}{c}\text { Porcentaje de } \\
\text { realización }\end{array}$ & $\begin{array}{c}\text { Duración } \\
\text { media }\end{array}$ \\
\hline $\begin{array}{c}\text { Tensión- } \\
\text { explosión }\end{array}$ & $48 \%$ & $123 \mathrm{~ms}$ & $30 \%$ & $113 \mathrm{~ms}$ & $39 \%$ & $118 \mathrm{~ms}$ \\
\hline $\begin{array}{l}\text { Tensión- } \\
\text { fricción }\end{array}$ & $28 \%$ & $109 \mathrm{~ms}$ & $39 \%$ & $97 \mathrm{~ms}$ & $33 \%$ & $103 \mathrm{~ms}$ \\
\hline Fricción & $24 \%$ & $78 \mathrm{~ms}$ & $31 \%$ & $63 \mathrm{~ms}$ & $28 \%$ & $70.5 \mathrm{~ms}$ \\
\hline
\end{tabular}


Asimismo, se observó que existe relación entre el porcentaje en el que se realizan los tipos de articulación y los rangos de duración propuestos por Martínez Celdrán (1993). En las figuras 1 y 2 se aprecia que la frecuencia de las realizaciones de tensión-explosión se relaciona con la duración del fonema, siendo más frecuente en el grupo de los sonidos geminados (141 ms o más). En cuanto a la fricción, el porcentaje de producciones es inversamente proporcional a la duración: son más frecuentes las realizaciones fricativas en el rango sonoro (menos de $69 \mathrm{~ms}$ ), mientras que en el geminado es prácticamente nulo. La tensión-fricción tiene mayor presencia en el rango sordo (70 a $140 \mathrm{~ms}$ ) donde alcanza el mayor porcentaje respecto a los otros dos rangos de duración. No obstante, la tensiónfricción no es la realización más frecuente en este rango, por lo que se podría interpretar que el rango sordo es, de hecho, una zona de transición en la que no se favorece ningún tipo de articulación en especial.

Figura 1. Tipos de articulación según los rangos de duración en la voz del edéntulo.

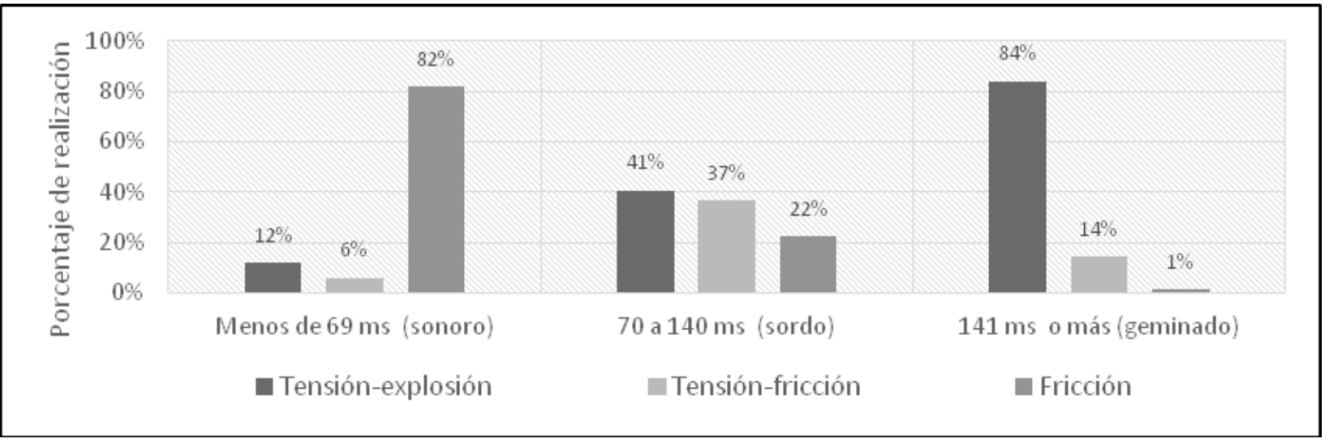

Figura 2. Tipos de articulación según los rangos de duración en la voz del portador de prótesis.

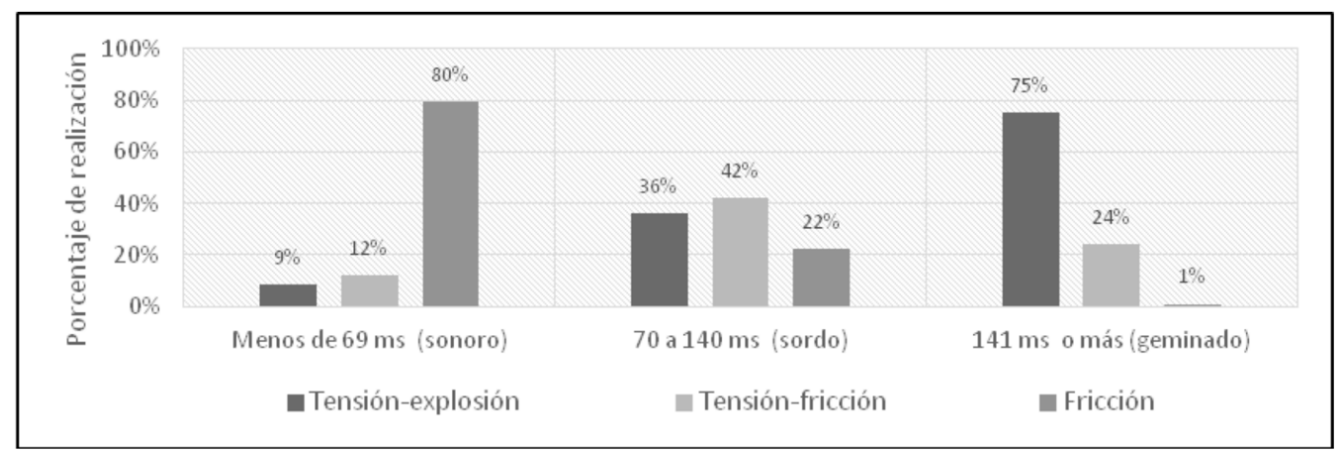




\subsubsection{Segunda fase articulatoria de /t/}

El análisis de la segunda fase articulatoria permitió observar que la duración media de esta fase fue de $32 \mathrm{~ms}$. En el caso de las realizaciones explosivas fue de $31 \mathrm{~ms} \mathrm{y}$ para las fricativas fue de $33 \mathrm{~ms}$. En el cuadro 4 se observa que el 54\% de las realizaciones corresponden a relajaciones explosivas, mientras que el $46 \%$ a fricativas. También se pudo comprobar que en la fonarticulación del adulto mayor edéntulo son más recurrentes las realizaciones explosivas, con un $63 \%$, en comparación con la articulación con prótesis, con un $43 \%$.

Con respecto a los rangos de duración de esta fase, al tomarse en cuenta el total de las realizaciones se pudo apreciar que el $62 \%$ se concentró en el rango medio, en tanto que el 21\% corresponde al breve, y el 17\% largo. Esto podría indicar que el área de contacto entre los órganos articuladores es más amplia que en las descripciones realizadas por otros fonetistas (Villamizar 2002; Martínez Celdrán y Fernández Planas 2007), quienes sostienen que la explosión en las oclusivas tiene una duración máxima de $10 \mathrm{~ms}$.

También se observó que en los casos en los que la oclusiva tenía una duración en el rango de los fonemas sonoros (menos de $69 \mathrm{~ms}$ ), no hubo realizaciones de la segunda fase que superaran los $40 \mathrm{~ms}$ de duración, es decir, no se presentaron casos con una segunda fase larga. Esto parece indicar que la segunda fase articulatoria no puede ser excesivamente larga si la fase oclusiva previa tiene una duración reducida.

Cuadro 4. Tipos de articulación de la 2a fase por rangos de duración

\begin{tabular}{|c|c|c|c|c|c|c|c|}
\hline & \multicolumn{2}{|c|}{ Edéntulo } & \multicolumn{2}{|c|}{ Prótesis } & \multicolumn{2}{|c|}{ Total } \\
\hline & & $\begin{array}{l}\text { Porcentaje } \\
\text { de } \\
\text { realización }\end{array}$ & $\begin{array}{c}\text { Duración } \\
\text { media en } \\
\text { ms }\end{array}$ & $\begin{array}{l}\text { Porcentaje } \\
\text { de } \\
\text { realización }\end{array}$ & $\begin{array}{c}\text { Duración } \\
\text { media en } \\
\text { ms }\end{array}$ & $\begin{array}{l}\text { Porcentaje } \\
\text { de } \\
\text { realización }\end{array}$ & $\begin{array}{l}\text { Duración } \\
\text { media en } \\
\text { ms }\end{array}$ \\
\hline \multirow{3}{*}{ Explosión } & Breve & $16 \%$ & 19.9 & $19 \%$ & 15.5 & $17 \%$ & 17.7 \\
\hline & Medio & $39 \%$ & 28.8 & $21 \%$ & 27.9 & $30 \%$ & 28.35 \\
\hline & Largo & $8 \%$ & 47.7 & $3 \%$ & 46.6 & $7 \%$ & 47.15 \\
\hline Total & & $63 \%$ & & $43 \%$ & & $54 \%$ & \\
\hline \multirow{3}{*}{ Fricción } & Breve & $1 \%$ & 19.4 & $7 \%$ & 16.6 & $4 \%$ & 18 \\
\hline & Medio & $25 \%$ & 31.1 & $40 \%$ & 31.7 & $32 \%$ & 31.4 \\
\hline & Largo & $11 \%$ & 50 & $10 \%$ & 49.3 & $10 \%$ & 49.65 \\
\hline Total & & $37 \%$ & & $57 \%$ & & $46 \%$ & \\
\hline
\end{tabular}




\subsubsection{Duración total de $|t|$}

En el corpus de estudio se encontraron realizaciones del fonema dental oclusivo sordo / $\mathrm{t}$ / con una duración total que iba desde los $23 \mathrm{~ms}$ hasta los $271 \mathrm{~ms}$, con una media de $93.1 \mathrm{~ms}$. Con ello se observa que los valores mínimos y máximos son muy distintos del rango de los fonemas oclusivos sordos señalados por Martínez Celdrán (1993).

Cuadro 5. Duración total del segmento en en el edéntulo y portador de prótesis

\begin{tabular}{|c|c|c|c|c|c|c|}
\hline & \multicolumn{2}{|c|}{ Edéntulo } & \multicolumn{2}{c|}{ Con prótesis } & \multicolumn{2}{c|}{ Total } \\
\cline { 2 - 7 } & $\begin{array}{c}\text { Porcentaje } \\
\text { de } \\
\text { realivación }\end{array}$ & $\begin{array}{c}\text { Duración } \\
\text { media en } \\
\text { ms }\end{array}$ & $\begin{array}{c}\text { Porcentaje } \\
\text { de } \\
\text { realivación }\end{array}$ & $\begin{array}{c}\text { Duración } \\
\text { media en } \\
\text { ms }\end{array}$ & $\begin{array}{c}\text { Porcentaje } \\
\text { de } \\
\text { realivación }\end{array}$ & $\begin{array}{c}\text { Duración } \\
\text { media en } \\
\text { ms }\end{array}$ \\
\hline Sonoro & $12 \%$ & 58.7 & $21 \%$ & 54.8 & $16.50 \%$ & 56.1 \\
\hline Sordo & $64 \%$ & 98.2 & $65 \%$ & 94.6 & $64.50 \%$ & 96.3 \\
\hline Geminado & $24 \%$ & 171.6 & $14 \%$ & 165.1 & $19.00 \%$ & 169.1 \\
\hline Media & - & 100.8 & - & 86.5 & - & 93.1 \\
\hline
\end{tabular}

Como se muestra en el cuadro 5, la duración es una de las características más estables en el corpus de estudio. La duración se mantiene en el rango de los fonemas sordos en el 64\% de los casos para la voz del adulto mayor edéntulo y en el 65\% para el portador de prótesis dentales. Solo se puede observar una diferencia si se toman en cuenta los rangos sonoro y geminado, en los que el edentulismo propicia realizaciones geminadas y las prótesis realizaciones sonoras. En el mismo sentido, se observa nuevamente que la falta de órganos dentarios propicia una mayor duración, con una media de 100.8 ms, en comparación con los 86.5 ms de los casos con prótesis.

\subsection{Resultados del análisis perceptivo}

\subsubsection{Test 001. Identificación de /t/ frente a /d/}

La principal finalidad de este test fue determinar si la duración total del estímulo es una característica que favorece la identificación de / $/$. Se tomaron en cuenta los tres rangos de duración que propone Martínez Celdrán (1993) para los fonemas oclusivos del español. Los resultados de este test mostraron que conforme aumenta la duración del segmento se favorece la correcta identificación del fonema /t/, tal como se muestra en la figura 3. 
Figura 3. Porcentaje de identificación según la duración del estímulo.

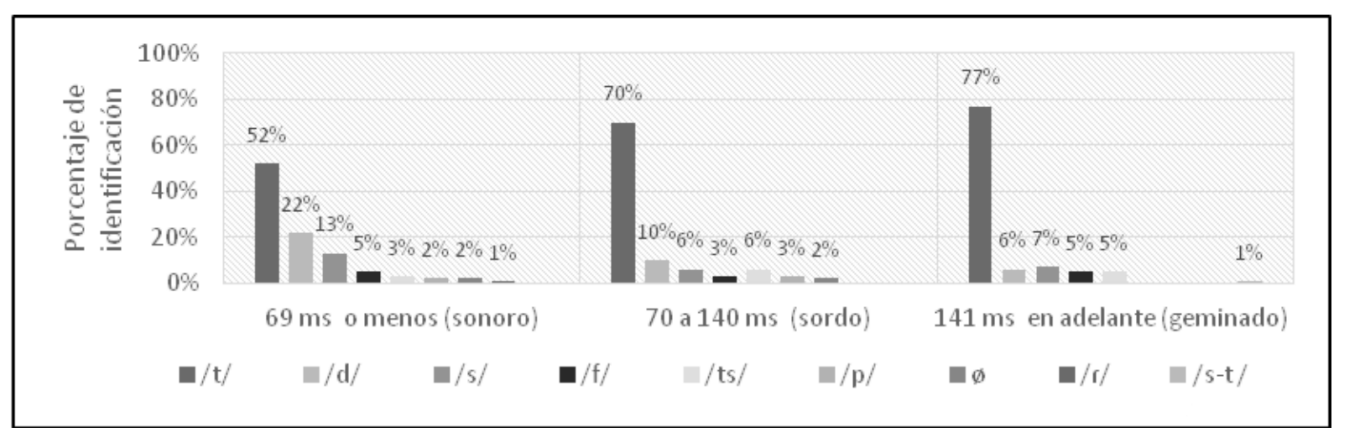

Destaca que en el rango de los fonemas sonoros se identifica hasta en un 52\% de los casos el fonema de estudio. Se esperaría que este resultado fuera mucho menor. Esto indica que, si bien la cantidad del estímulo aporta cierta información sobre el tipo de oclusiva, existen otras características acústicas que intervienen en la distinción entre /d-t/.

Los estímulos fueron identificados en unos pocos casos como fonemas distintos a los oclusivos. El 18\% de los estímulos se percibió como un fonema fricativo: /s/ o /f/; el 3\% como un fonema africado: /ts/; y el 5\% restante como algún otro tipo de fonema o sonido. Esto indica que la duración del estímulo sí aporta información suficiente para la distinción entre obstruyentes oclusivas, fricativas y africadas, pero no otorga información concluyente sobre la sonoridad.

Otro resultado relevante que se observó fue que en ningún caso se identificó un fonema geminado, ni siquiera en el rango de duración superior a los $141 \mathrm{~ms}$. Esto quizás se deba a que los oyentes no discriminaron entre / $\mathrm{t} / \mathrm{y} / \mathrm{t}$ :/, pues la segunda no tiene un valor fonológico en el español.

\subsubsection{Test 002. Discriminación de /t/ frente a /d/}

Este test permitió comprobar los resultados del test 001, ya que se observó que la duración del estímulo aporta información sobre la categoría a la que pertenece la oclusiva. Una de las primeras diferencias entre este test y el anterior es que sí se realiza la distinción de /t/ y /t:/, debido a que las instrucciones del experimento sí lo permitían. Además, en la figura 4 se observa que la identificación de /t/ en el rango sonoro es muy alta, del 62\%, lo que nuevamente indica que la duración del fonema no aporta información suficiente para distinguir la sonoridad de este. 
Figura 4. Porcentaje de discriminación de /t/ frente a /d/.

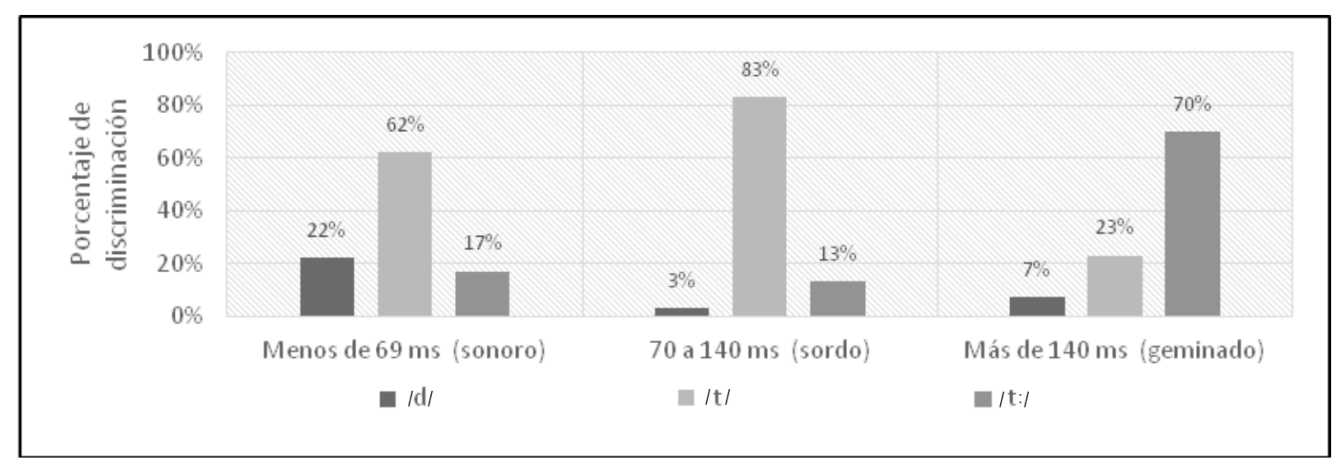

\subsubsection{Test 003. Identificación de /t/ frente a /t $\mathrm{tl}$}

El objetivo en este test fue comprobar la importancia de la segunda fase articulatoria en la identificación del fonema de estudio. Fueron considerados los rangos de duración propuestos para la segunda fase articulatoria: $20 \mathrm{~ms}$ o menos corresponde al rango breve, de $21 \mathrm{~ms}$ a $40 \mathrm{~ms}$ al rango medio, y $41 \mathrm{~ms}$ o más al rango largo. Además de la duración, se comprobó si el tipo de articulación, oclusivo o fricativo, tenía alguna función en la identificación de / $\mathrm{t} /$.

Los resultados obtenidos muestran que la duración de la segunda fase interviene directamente en la correcta identificación del fonema de estudio. El porcentaje de correcta identificación disminuye conforme aumenta la duración de esta fase, hasta llegar a solo un $30 \%$ de identificación en el rango largo (véase la figura 5). A partir de este test se obtienen dos categorías según la duración de esta fase articulatoria: la primera está constituida por aquellos casos en los que se identifica /t/ y cuya duración es menor a $40 \mathrm{~ms}$. La segunda está conformada por los casos en los que esta fase presenta una duración mayor a $41 \mathrm{~ms}$, y en los que no se identifica ningún fonema mayoritariamente. 
Figura 5. Porcentaje de identificación de estímulos según la duración de la 2a fase articulatoria.

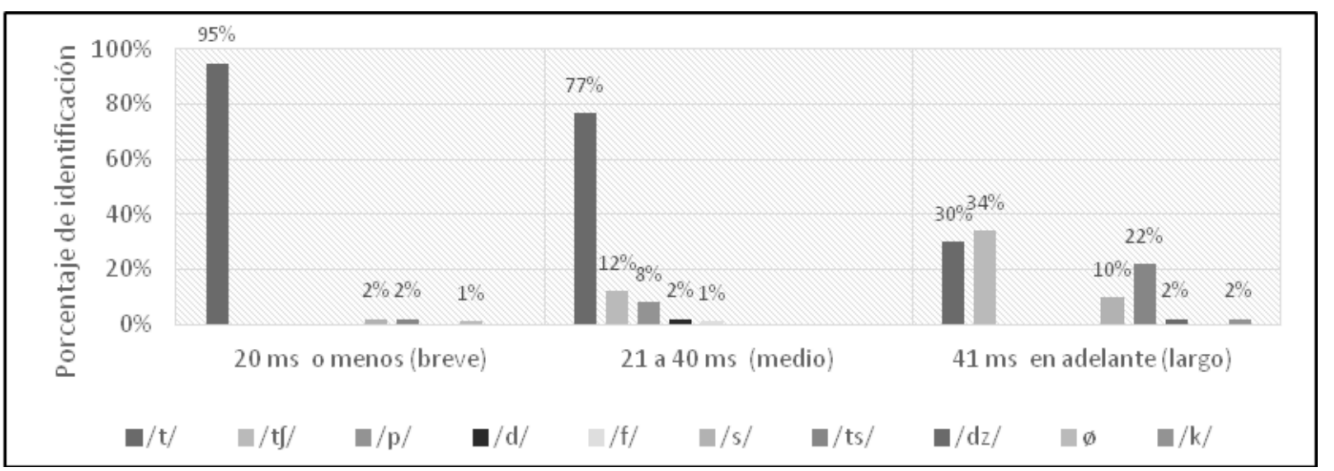

Algo que es interesante destacar es que en el último rango, como se observa en la figura 5, se presenta mucha confusión en la identificación del estímulo, ya que no solo se dificultó la identificación de /t/, sino que también se confundió con otros fonemas como el palatal africado $/ \mathrm{t} /$ o el prepalatal africado sordo /ts/, principalmente. Esto indica que una duración superior a los $41 \mathrm{~ms}$ genera confusión en el oyente, mientras que, en los rangos medio y breve, menores a $40 \mathrm{~ms}$, el oyente muestra un porcentaje de identificación mayoritario de $/ \mathrm{t} /$.

Cuadro 6. Identificación de obstruyentes según la duración y realización de la 2a fase

\begin{tabular}{|c|c|c|c|c|}
\hline $\begin{array}{c}\text { Modo de } \\
\text { articulación }\end{array}$ & $\begin{array}{c}\text { Tipo de } \\
\text { obstruyente }\end{array}$ & $\begin{array}{c}\mathbf{2 0} \text { ms o menos } \\
\text { (breve) }\end{array}$ & $\begin{array}{c}\mathbf{2 1} \text { a } \mathbf{4 0} \text { ms } \\
\text { (medio) }\end{array}$ & $\begin{array}{c}41 \text { ms o más } \\
\text { (largo) }\end{array}$ \\
\hline \multirow{4}{*}{ Explosión } & Oclusivo & $93 \%$ & $97 \%$ & $33 \%$ \\
\cline { 2 - 5 } & Fricativo & $3 \%$ & $3 \%$ & $4 \%$ \\
\cline { 2 - 5 } & Africado & - & - & $63 \%$ \\
\cline { 2 - 5 } & Otro & $4 \%$ & - & - \\
\hline \multirow{4}{*}{ Fricción } & Oclusivo & $97 \%$ & $76 \%$ & $20 \%$ \\
\cline { 2 - 5 } & Fricativo & - & - & $50 \%$ \\
\cline { 2 - 5 } & Africado & $3 \%$ & $24 \%$ & - \\
\cline { 2 - 5 } & Otro & - & - & $30 \%$ \\
\hline
\end{tabular}


Al considerarse el tipo de articulación de la segunda fase se encontró que, en el rango breve, es indistinto el modo de articulación para la identificación de los fonemas oclusivos, tal como se muestra en el cuadro 6. Esto puede indicar que es más importante la duración de esta fase que el modo en el que se produce. En el rango medio de 21 a 40 ms aún se identifican con facilidad los fonemas oclusivos, aunque en la articulación fricativa se puede confundir hasta en el $24 \%$ de los casos con los fonemas africados. En el rango largo se puede apreciar un alto grado de indecisión en la identificación del tipo de fonema. Se observa que en la realización explosiva se favorece la identificación de fonemas africados, lo cual llama la atención, pues se esperaría que esta tendencia fuera característica de las realizaciones fricativas.

\subsubsection{Test 004. Discriminación de /t/ frente a $|t|$}

Los resultados del test de discriminación permitieron confirmar que en el rango de duración superior a los $41 \mathrm{~ms}$ la identificación de / $/$ / disminuye considerablemente, aunque no con un porcentaje tan bajo como en el test 003 (véase la figura 6). Se confirman, por tanto, las dos categorías observadas en el test 003: $40 \mathrm{~ms}$ o menos permiten la identificación de / $\mathrm{t} /$, en tanto que una duración de más de $41 \mathrm{~ms}$ disminuye la correcta identificación de este fonema.

Figura 6. Porcentaje de discriminación de /t/ frente a $/ \mathrm{t} \int /$.

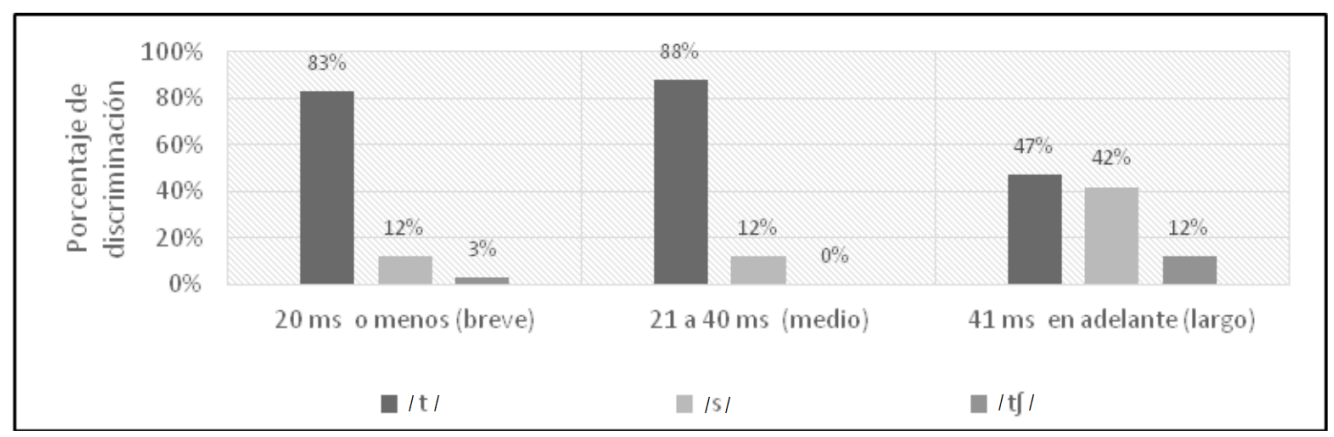

La identificación de los fonemas africados fue muy baja si se le compara con los resultados del test 003 , en el que estos fonemas llegaron a tener hasta el 58\% de identificación en el rango largo. En el test 004, el rango largo favoreció la identificación del fonema /s/ (42\%) por encima del / $\mathrm{t} / \mathrm{f}(12 \%)$. Esto resulta peculiar ya que el estímulo tenía características más cercanas a $/ \mathrm{t} \mathrm{f} /$, una fase tensa y una segunda fase con una duración mayor a $41 \mathrm{~ms}$ con articulación fricativa.

En el cuadro 7 se observa que existen resultados distintos a los encontrados en el test 003 con respecto a la identificación de /t/, ya que la tendencia observada de que, 
a mayor duración de la segunda fase, menor es la identificación de /t/, no se cumple en la articulación explosiva. En el caso de la articulación fricativa sí se cumple la tendencia descrita, pues únicamente se identifica/t/ hasta en el $23 \%$ de los casos.

Cuadro 7. Discriminación de /t/ según la duración y modo de articulación de la segunda fase

\begin{tabular}{|c|c|c|c|c|}
\hline $\begin{array}{c}\text { Modo de } \\
\text { articulación }\end{array}$ & $\begin{array}{c}\text { Tipo de } \\
\text { obstruyente }\end{array}$ & $\begin{array}{c}20 \text { ms o menos } \\
\text { (breve) }\end{array}$ & $\begin{array}{c}21 \text { a } \mathbf{4 0} \text { ms } \\
\text { (medio) }\end{array}$ & $\begin{array}{c}41 \text { ms o más } \\
\text { (largo) }\end{array}$ \\
\hline \multirow{3}{*}{ Explosión } & $/ \mathrm{t} /$ & $74 \%$ & $97 \%$ & $70 \%$ \\
\cline { 2 - 5 } & $/ \mathrm{s} /$ & $20 \%$ & - & $24 \%$ \\
\hline \multirow{3}{*}{ Fricción } & $/ \mathrm{t} /$ & $6 \%$ & $3 \%$ & $6 \%$ \\
\cline { 2 - 5 } & $/ \mathrm{t} /$ & $94 \%$ & $80 \%$ & $23 \%$ \\
\cline { 2 - 5 } & $/ \mathrm{s} /$ & $6 \%$ & - & $60 \%$ \\
\hline
\end{tabular}

\subsubsection{Test 005. Identificación de /t/ frente a $/ \mathrm{s} /$}

La realización fricativa de /t/ fue uno de los resultados más interesantes en el análisis acústico, pues no cuenta con ninguna de las características acústicas prototípicas de los fonemas oclusivos y se observó que el porcentaje de realizaciones estaba relacionado con la duración del segmento. Este test permitió determinar si dicha relación tiene una base perceptiva. Los estímulos están constituidos por realizaciones fricativas de $/ \mathrm{t} / \mathrm{con}$ duraciones en los tres rangos de duración que propone Martínez Celdrán (1993).

Figura 7. Porcentaje de identificación de /t/ según la duración de la realización fricativa.

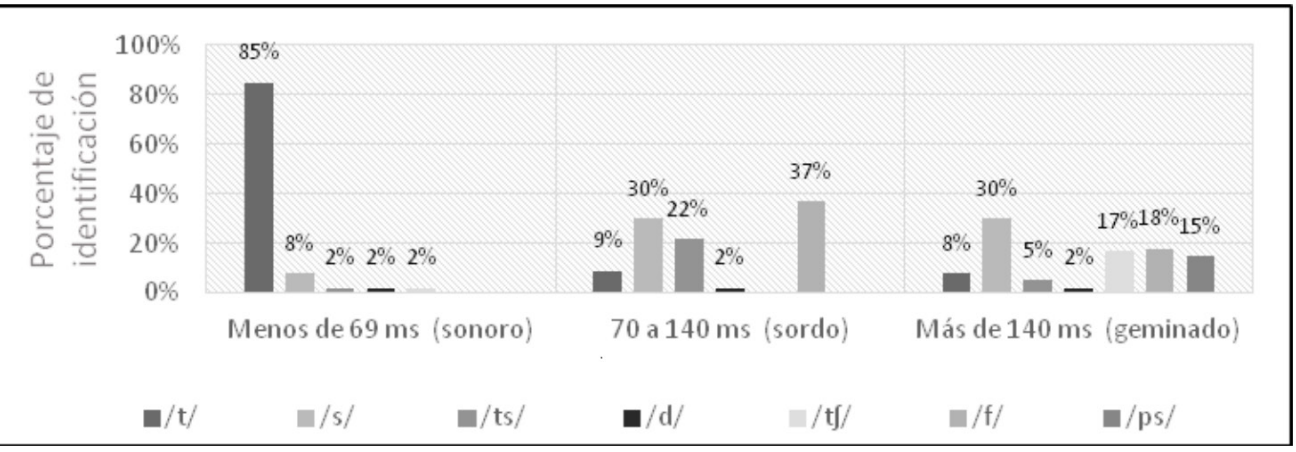


Los resultados de este test mostraron que el fonema / $\mathrm{t} /$ con realización fricativa se identifica correctamente solo en los estímulos en los que la duración se reduce hasta el rango de los fonemas sonoros, tal como se muestra en la figura 7 . Si presenta una duración en el rango sordo o geminado se confunde con distintos fonemas. En el cuadro 8 se aprecia que, en el rango de los sonidos sordos, un mayor porcentaje de estímulos se percibe como fonemas fricativos; mientras que, en el rango geminado, el porcentaje de percepción es muy similar entre fonemas fricativos y africados.

Cuadro 8. Tipo de fonema identificado según la duración del fonema

\begin{tabular}{|c|c|c|c|}
\hline $\begin{array}{c}\text { Tipo de fonema } \\
\text { identificado }\end{array}$ & $\begin{array}{c}\text { Menos de 69 ms } \\
\text { (sonoro) }\end{array}$ & $\begin{array}{c}70 \text { a 140 ms } \\
\text { (sordo) }\end{array}$ & $\begin{array}{c}\text { Más de 140 ms } \\
\text { (geminado) }\end{array}$ \\
\hline Oclusivo & $87 \%$ & $11 \%$ & $12 \%$ \\
\hline Fricativo & $8 \%$ & $68 \%$ & $49 \%$ \\
\hline Africado & $5 \%$ & $22 \%$ & $38 \%$ \\
\hline
\end{tabular}

\subsubsection{Test 006. Discriminación de $|t|$ frente a $/ s \mid$}

Al igual que en el test 005 , los estímulos constituyen realizaciones fricativas del fonema $/ \mathrm{t} /$ con duraciones en los rangos sonoro, sordo y geminado. Los resultados de este test permitieron comprobar que a menor duración se favorece la identificación de $/ \mathrm{t} /$. La diferencia principal con respecto al test anterior es que existe una disminución gradual en la identificación, de forma que se identifica en el $88 \%$ de los casos en el rango sonoro, $44 \%$ en el rango sordo y $23 \%$ en el geminado, tal como se aprecia en la figura 8.

Figura 8. Porcentaje de discriminación de /t/ según la duración de la realización fricativa.

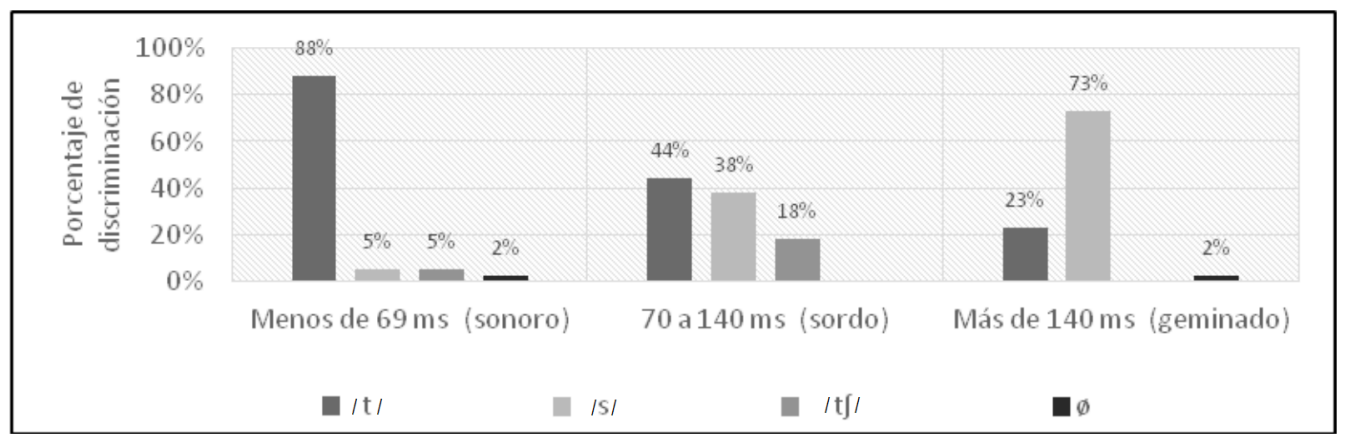


Con base en los resultados del test 006, se aprecia una relación entre la cantidad de realizaciones fricativas que se produjeron en el corpus de estudio y el porcentaje de identificación auditiva de este tipo de articulación según la duración. Por un lado, se observó en la figura 1 que conforme aumenta la duración del estímulo, disminuye el porcentaje de realizaciones fricativas. Por otro lado, en la figura 8 se observa una tendencia similar, en la que, al aumentar la duración del estímulo, disminuye el porcentaje de identificación del fonema cuando tiene una realización fricativa. Esto podría indicar que la disminución de las realizaciones fricativas conforme aumenta la duración tiene una motivación perceptiva.

\section{DisCUSIÓN Y CONCLUSIONES}

Los resultados permitieron confirmar que la producción del fonema /t/ tiene una alta variabilidad en el corpus de estudio, y que la articulación prototípica: oclusiva con explosión breve y en el rango sordo, representa un bajo porcentaje de las realizaciones de / $\mathrm{t} /$. Esto debido a los cambios anatómicos en la cavidad oral de los adultos mayores edéntulos y portadores de prótesis dentales.

Además, el uso de prótesis dentales genera cambios en la producción de este fonema en comparación con la del adulto mayor edéntulo. La cantidad de articulaciones oclusivas explosivas en edéntulos es considerablemente mayor a la presentada en las realizaciones con prótesis. El uso de prótesis motiva las fricciones, mientras que el edentulismo propicia una segunda fase explosiva. Esto debido a que el fonema /t/ únicamente necesita del borde de los alveolos para articularse de forma prototípica. Martínez Celdrán y Fernández Planas (2007) informan que, normalmente, este fonema se produce tanto en la cara anterior de los incisivos superiores como en el límite de los alveolos. Además, en el habla del edéntulo, el entorno de la cavidad oral no sufre otro tipo de modificaciones importantes, tales como la disminución del área de la cavidad oral.

La alta cantidad de articulaciones con elementos fricativos en el habla de los portadores de prótesis dentales totales puede tener un origen articulatorio multifactorial: la falta de rugas palatinas (Kong y Hansen 2008), el grosor de la plancha acrílica (Martínez Contreras 2013) o la disminución del volumen de la cavidad oral.

Otro hallazgo fue que la duración de las realizaciones de /t/ es una de las características más estables en el corpus de estudio, ya que, en su mayoría, se mantiene en el rango de los fonemas sordos, tanto para la voz del adulto mayor edéntulo como la del portador de prótesis dentales.

Los resultados de los test perceptivos confirmaron que el fonema dental oclusivo sordo /t/ se percibe como tal aún cuando existen cambios en sus características acústicas prototípicas.

La duración total del estímulo es una característica que favorece la identificación de /t/. Conforme aumenta la duración del segmento se favorece la correcta identificación del fonema / $/$ / No obstante, al existir también un alto porcentaje de identificación en el 
rango de los fonemas sonoros, se concluye que si bien la cantidad del estímulo aporta cierta información sobre el tipo de oclusiva, existen otras características acústicas que intervienen en la distinción entre /d-t/. Es decir, la duración del estímulo sí aporta información suficiente para la distinción entre obstruyentes oclusivas, fricativas y africadas, pero no otorga información concluyente sobre la sonoridad.

Respecto a la pertinencia de la duración de la segunda fase articulatoria en la identificación del fonema de estudio se encontró que el porcentaje de correcta identificación disminuye conforme aumenta la duración de esta fase, solo en el caso de la articulación fricativa y no en el de la explosiva. Y que al considerarse el tipo de articulación de la segunda fase, en el rango breve es indistinto el modo de articulación para la identificación de los fonemas oclusivos. Esto puede indicar que es más importante la duración de esta fase que el modo en el que se produce.

Ahora bien, la realización fricativa de /t/ que se encontró en el análisis acústico no cuenta con ninguna de las características acústicas prototípicas de los fonemas oclusivos y se observó que el porcentaje de realizaciones estaba relacionado con la duración del segmento. El test perceptivo permitió determinar su relación con una base perceptiva, pues esta realización fricativa se identifica correctamente solo en los estímulos en los que la duración se reduce hasta el rango de los fonemas sonoros (menores a $69 \mathrm{~ms}$ ). Es decir, que a menor duración se favorece la identificación de $/ \mathrm{t} /$.

Existen estudios en los que se ha observado que entre más posterior sea la consonante, mayor es la duración de la fase explosiva, lo que podría ser un proceso análogo en el caso de la fricción. En el cuadro 9 se aprecia el rasgo que corresponde a cada una de las dos categorías creadas a partir de la clasificación de los estímulos con duración menor a 69 $\mathrm{ms}$ y mayor a $70 \mathrm{~ms}$ por diferentes autores.

Cuadro 9. Rasgos según la duración total del segmento en la articulación fricativa de /t/

\begin{tabular}{|c|c|c|}
\hline Tipo de rasgo & $\begin{array}{c}\text { Duración total del fonema } \\
\text { menor a } 69 \mathbf{~ m s}\end{array}$ & $\begin{array}{c}\text { Duración total del fonema } \\
\text { mayor a 70 ms }\end{array}$ \\
\hline $\begin{array}{c}\text { Rasgos articulatorios } \\
\text { (Quilis, 1999) }\end{array}$ & [alveolar] \\
\hline $\begin{array}{c}\text { Rasgos acústicos } \\
\text { (Quilis, 1999) }\end{array}$ & {$[$ Mate] } & [Estridente] \\
\hline $\begin{array}{c}\text { Rasgos articulatorios } \\
\text { (RAE y AALE, 2011) }\end{array}$ & $\begin{array}{c}\text { [-estridente] } \\
\text { [-distribuido] }\end{array}$ & $\begin{array}{c}\text { [+estridente] } \\
\text { [+distribuido] }\end{array}$ \\
\hline $\begin{array}{c}\text { Martínez Celdrán } \\
(1984)\end{array}$ & {$[$ [tojo] } & [tenso] \\
\hline
\end{tabular}


Llama la atención que la única diferencia entre la realización fricativa de /t/ y el fonema /s/ en el plano acústico es la duración y la intensidad con la que se realiza la fricción. Esto lleva a pensar que el rasgo que genera la oposición entre dichos sonidos es el rasgo [tenso-flojo]. Martínez Celdrán (1984) propone que este rasgo no solo permite la distinción entre fonemas oclusivos, sino entre tipos de obstruyentes.

En los resultados también se cumple con el carácter gradual de este rasgo, pues al igual que en los resultados de Liberman et al. (1961), se aprecia que en el rango menor a 69 ms se identifica/t/ hasta en el $87 \%$ de los casos; en el rango superior a los $140 \mathrm{~ms}$ se identifica el fonema $/ \mathrm{s} /$ en un $72 \%$ de los casos; mientras que el rango intermedio muestra una zona de transición entre las dos categorías puesto que el porcentaje de identificación de /t/ y /s/ resulta similar (véase la figura 9).

Figura 9. Función del rasgo [tenso-flojo] en la identificación de /t/ y /s/.

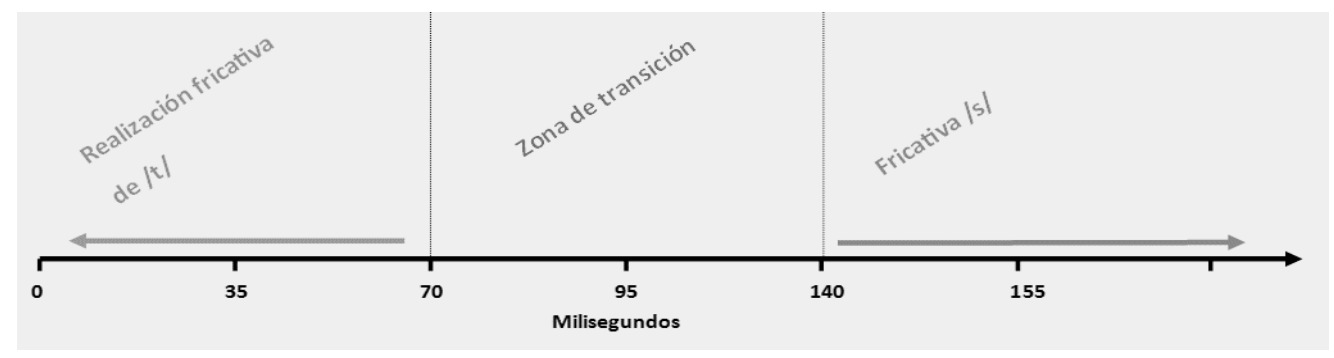

El principal problema de definir la oposición entre la realización fricativa de /t/ y el fonema $/ \mathrm{s} /$ mediante el rasgo [tenso-flojo] sería que el fonema / $\mathrm{t} /$ tendría que caracterizarse con el rasgo [flojo], lo cual contradice la caracterización prototípica del fonema respecto a su rasgo [tenso]. Por ello, se tiene que tomar en cuenta que esta es una de las realizaciones de /t/ que no conserva ninguna de sus características prototípicas: la duración, la fase tensa y la explosión; únicamente conserva el carácter sordo del fonema. Lo interesante es que a pesar de ello la realización fricativa de / $/$ / se identifica correctamente en el rango de duración de las sonoras.

Las aproximaciones realizadas sobre los efectos del edentulismo y las prótesis dentales en la articulación de / $/$ / se han basado en los efectos acústicos observados, tales como la duración de la segunda fase articulatoria, el modo de articulación y la duración general del segmento; no obstante, dados los alcances del presente estudio, no se ha contado con una observación directa de los procesos articulatorios realizados, y por tanto, no se ha tenido una descripción apoyada en un correlato fisiológico del rasgo [tenso-flojo].

Con los hallazgos aquí presentados se plantea la posibilidad de realizar un estudio del fonema /t/ en todos los contextos fónicos, con la finalidad de validar los resultados obtenidos en la presente investigación. Además, resulta pertinente profundizar en el estudio 
de los casos en los que el fonema fue identificado aun cuando los cambios acústicos fueron muy grandes. Dicho estudio tendría una postura puramente fonológica.

\section{Obras citadas}

Álvarez, Juan Antonio. 1978. "El análisis por síntesis". Revista española de lingüistica 8.1: 117-124.

Ibabe, Alexander, Petrirena Rosa \& Aguirrezabal Itziar. 2019. "La articulación de las consonantes velares del español”. Estudios de fonética experimental, 125-160.

Kong, Hyung-Jun \& Hansen Carl. 2008. "Customizing palatal contours of a denture to improve speech intelligibility”. The Journal of Prosthetic Dentistry 99.3: 243-248.

Liberman, Alvin, Harris Katherine, Eimas Peter, Lisker Leigh \& Bastian Jarvis. 1961. "An effect of learning on speech perception: the discrimination of durations of silence with and without phonemic significance". Language and Speech 4.4: 175-195.

Lisker, Leigh. 1957. "Closure duration and the intervocalic voiced-voiceless distinction in English". Language 33: 42-49.

Martínez Celdrán, Eugenio. 1984. "Cantidad e intensidad en los sonidos obstruyentes del castellano. Hacia una caracterización acústica de los sonidos aproximantes”. Estudios de fonética experimental 1: 71-129.

. 1991. "Relevancia de los elementos de las oclusivas sordas del castellano según la discriminación auditiva”. Fonética experimental: Teoría y práctica. Madrid, España: Síntesis. . 1993. "La percepción categorial de /b-p/ en español basada en las diferencias de duración”. Estudios de fonética experimental 5: 223-239.

Martínez Celdrán, Eugenio \& Fernández Planas Ana María. 2007. Manual de fonética española. Barcelona, España: Ariel.

Martínez Contreras, César. 2013. "Factores que intervienen en la fonética del paciente portador de prótesis dental removible". Tesis de pregrado. Ciudad de México, México: Universidad Nacional Autónoma de México.

Martínez, Hernán, Mora Elsa \& Prato Rafael. 2006. “Influencia de los aparatos dentales ortodónticos en la producción y percepción de habla: estudio de cinco casos". Revista CEFAC 8.4: 467-476.

Planas, Ana María, Ibabe Alexander, Petrirena Rosa \& Aguirrezabal Itziar. 2020. "La tensión articulatoria de las obstruyentes oclusivas en español". Estudios de fonética experimental, 91-123.

Quilis, Antonio. 1999. Tratado de fonología y fonética españolas (2da edic.). Madrid, España: Gredos.

Real Academia Española \& de la Lengua, A. D. A. 2011. Nueva Gramática de la Lengua Española. Fonética y Fonología. Barcelona, España: Espasa.

Runte, Christoph, Lewerino Michael, Dirksen Dieter, Bollmann Friedhelm, LamprechtDinnesen Antoinette \& Seifert Eberhard. 2001. "The influence of maxillary central 
incisor position in complete dentures on /s/ sound production". Journal of Prosthetic Dentistry 85.5: 485-495.

Seifert, Eberhard, Runte Christoph, Riebandt Michael, Lamprecht-Dinnesen Antoinette \& Bollmann Friedhelm. 1999. "Can dental prostheses influence vocal parameters?". Journal of Prosthetic Dentistry, 81.5: 579-585.

Villamizar, Thania. 2002. "Caracterización acústica de las consonantes oclusivas en el español de Venezuela. El parámetro duración (silencio-barra de explosión-VOT)”. Lengua y Habla 7.1: 140-157. 